\title{
A Numerical Approach to the Kinematic Analysis of Deployable Structures forming a Closed Loop
}

\author{
W.W. Gan and S. Pellegrino \\ Department of Engineering, University of Cambridge \\ Trumpington Street, Cambridge, CB2 1PZ, UK. \\ pellegrino@eng.cam.ac.uk
}

March 20, 2006

Keywords: Deployable structures, kinematic analysis, loop-closure.

\begin{abstract}
This paper is concerned with spatial linkages forming a closed loop. In one extreme configuration (deployed), these linkages form a frame of polygonal shape, such as a square or a hexagon, and in the other extreme (folded) configuration form a tight bundle. Throughout their motion range, they have mobility one. These linkages have potential applications for next-generation deployable spacecraft structures. The paper presents a systematic study of the kinematics of closed-loop structures with these special properties, and presents a numerical scheme for simulating their deployment without making any assumptions about particular symmetry features. The proposed simulation technique is applied to three examples that show different behaviour during deployment.
\end{abstract}

\section{Introduction and Background}

This paper is concerned with the kinematics of deployable structures, based on the concept of a mechanical linkage consisting of straight rods connected by revolute joints, that form a segmented hoop that folds into a tight bundle. A structure of this kind was proposed by J.M. Hedgepeth, following a preliminary suggestion by A. Busemann [1]. The idea was presented in 
reference [2], which deals mostly with a related structural concept, that was later adopted for the edge beam of the Hoop-Column Antenna shown in Figure 1. Here the rim consists of four or more hinged segments of equal-length, where each joint involves two revolutes connected by an intermediate short element. Even for hoops with a small number of segments, the presence of these blocks increases the mobility and hence, since kinematic freedom can lead to deployment uncertainty, in the Hoop-Column Antenna additional links were introduced to bring the mobility down to one.

Of particular interest to this paper is Appendix A of Reference [2], which considered the alternative hinging method, illustrated in Figure 2, where there is only one revolute between consecutive links. Note that the orientation of each revolute varies during deployment of the 10-sided linkage shown (unlike the Hoop-Column Antenna), and also note that 5-fold symmetry about a central axis has been imposed here.

An advantage of this particular hinging method is that it requires half the number of revolutes as the previous scheme and so the need for additional synchronization links is much reduced. Actually, the four-sided version of this linkage is an example of the classical 4-bar linkage discovered by G.T. Bennett [3], and the six-sided version had been discovered by Bricard (see the recent survey in Reference [4]). However, the particular realization of these linkages that was first proposed by Hedgepeth is the key to their practical exploitation as deployable structures.

Structures of this kind have potential applications for deployable spacecraft structures, in solar arrays — as already envisaged in Ref. [2] -, solar sails, and synthetic aperture antennas or radar. For example, a configuration that has been recently investigated in Reference [5] is the deployable rectangular frame shown in Figure 3.

It should be noted that spatial linkages based on the concept of a ring pantograph, which can itself be viewed as a structure consisting of multiple closed loops, have already been extensively studied and have found many applications $[6,7]$.

This paper presents a general approach for analysing the kinematics of closed-loop structures, without making any assumptions about their behaviour being symmetric. The proposed approach is particularly suitable to detect points of bifurcation along the motion path of such structures. The paper is arranged as follows. Section 2 introduces the notation and briefly outlines the kinematic formulation. Section 3 details the geometry of the closed-loop linkages proposed by Hedgepeth and derives the so-called loop-closure equation for these structures. For the case of 4-rod linkages, assuming symmetry, an analytical expression for the variation of the 
joint angles is obtained. Section 4 presents a novel numerical scheme for simulating the motion of a structure with mobility one, by carrying out an incremental solution of the loop closure equation. This scheme begins by imposing an initial set of angle changes on the revolutes; these changes have magnitudes proportional to the zero-energy mechanism of the structure, obtained from the singular-value decomposition of the (rank deficient) Jacobian matrix of the system. Next, an iterative correction step is carried out, to make the violation of the constraint equations smaller than a specified value. Section 5 presents the simulation results obtained from two of Hedgepeth's linkages and Section 6 then presents an additional set of results, for an alternative linkage that —unlike the previous examples - has a point of kinematic bifurcation along its motion path. Section 7 concludes the paper.

\section{Kinematic Formulation}

This section introduces the general description of geometric transformations of a cartesian coordinate system used in this paper. More details can be found in standard textooks $[\mathbf{8 , 9}]$.

The general position and orientation of a coordinate system, $P_{1}, x_{1}, y_{1}, z_{1}$, with respect to a fixed coordinate system, $O, X, Y, Z$, can be described by the translation from $O$ to $P_{1}$ followed by the rotation of $O, X, Y, Z$ to $P_{1}, x_{1}, y_{1}, z_{1}$, Figure 4 . This transformation is described by a vector $v_{1}$ $(3 \times 1)$ and a matrix $R_{1}(3 \times 3)$. The Denavit-Hartenberg notation [10] is adopted and hence

$$
T_{1}=\left[\begin{array}{cccc} 
& & & \\
& R_{1} & & v_{1} \\
& & & \\
0 & 0 & 0 & 1
\end{array}\right]
$$

where $R_{1}$ transforms $x_{1}, y_{1}, z_{1}$ into the global coordinate axes and is defined in terms of the Euler angles $\phi, \omega, \psi$ (we use the $x$-convention [11]). Hence,

$$
R_{1}=\left[\begin{array}{ccc}
\cos \phi & -\sin \phi & 0 \\
\sin \phi & \cos \phi & 0 \\
0 & 0 & 1
\end{array}\right]\left[\begin{array}{ccc}
1 & 0 & 0 \\
0 & \cos \omega & -\sin \omega \\
0 & \sin \omega & \cos \omega
\end{array}\right]\left[\begin{array}{ccc}
\cos \psi & -\sin \psi & 0 \\
\sin \psi & \cos \psi & 0 \\
0 & 0 & 1
\end{array}\right]
$$

The transformation from $P_{1}, x_{1}, y_{1}, z_{1}$ to $P_{2}, x_{2}, y_{2}, z_{2}$, shown in Figure 4 , is represented by

$$
T_{2}=\left[\begin{array}{cccc}
R_{2} & & v_{2} \\
& & & \\
0 & 0 & 0 & 1
\end{array}\right]
$$


where $v_{2}=P_{1} P_{2}$ and $R_{2}$, whose components are defined with respect to $x_{1}, y_{1}, z_{1}$, rotate $x_{2}, y_{2}, z_{2}$ into $x_{1}^{\prime}, y_{1}^{\prime}, z_{1}^{\prime}$. Note that $R_{2}$ goes in the opposite direction to $v_{2}$.

Consider the compound transformation $T_{1,2}$, i.e. $T_{1}$ followed by $T_{2}$. The translation vector is $v_{1}$ followed by $R_{1} v_{2}$ (as the second translation has to be expressed with respect to the first coordinate system), hence

$$
v_{1,2}=v_{1}+R_{1} v_{2}
$$

The rotation matrix is obtained from

$$
X=R_{1} x_{1}=R_{1} R_{2} x_{2}
$$

Therefore

$$
T_{1,2}=\left[\begin{array}{cccc}
R_{1} R_{2} & & v_{1}+R_{1} v_{2} \\
0 & 0 & 0 & 1
\end{array}\right]=\left[\begin{array}{cccc}
R_{1} & & v_{1} \\
& & & \\
0 & 0 & 0 & 1
\end{array}\right]\left[\begin{array}{cccc}
R_{2} & & v_{2} \\
& & & \\
0 & 0 & 0 & 1
\end{array}\right]=T_{1} \times T_{2}
$$

A more general compound transformation can be expressed as

$$
T_{1, n}=T_{1} \times T_{2} \times \ldots \times T_{n}
$$

\section{$3 \quad$ Hedgepeth's Deployable Structures}

Hedgepeth [3] proposed a family of closed-loop deployable structures consisting of $n$ rods with identical cross section, where $n$ is even. The geometry of these structures is most easily described if the rod's cross-section is assumed to be an isosceles triangle; this is the case shown in Figure 2 and Figure 5. Note that more general rod shapes are acceptable, provided that the hinges can still be mounted in the required orientation and the rods do not interfere with the motion of the structure.

First, consider the structure in the configuration where it forms a tight bundle. For the rods to fit together in this configuration the apex angle of the isosceles triangles must be, see Figure 5(a),

$$
\alpha_{1}=\frac{360^{\circ}}{n}
$$

Hence, the base angles are

$$
\alpha_{2}=90^{\circ}-\frac{\alpha_{1}}{2}
$$


To ensure that the rods fit together also in the deployed configuration their ends are cut at an angle of $180^{\circ} / n$ to the axis of the rod and "door hinges" are mounted alternately on inner- and outer-facing faces of the rods, see Figure 5(b). The end sections of the rods are also isosceles triangles, with base angles

$$
\alpha_{3}=\arctan \left(\frac{\cos ^{2}\left(\alpha_{1} / 2\right)}{\sin \alpha_{1} / 2}\right)
$$

We describe each rod by means of a transformation matrix between two suitably defined coordinate systems, one at each end of the rod. The orientation of each coordinate system is such that the $z$-axis is aligned with the corresponding hinge axis and the line defined by the apexes of the isosceles triangles lies in the $x-z$ plane.

Figure 6 shows a sequence of four elementary transformations. First, Figure 6(a), one translates $O, X, Y, Z$ to $P_{1}, x, y, z$ by the vector $v_{1}$. Next, Figure $6(\mathrm{~b})$, one rotates $P_{1}, x, y, z$ about $z$ through $\phi$ to obtain $P_{1}, \xi, \eta, \zeta ; \phi$ is such that the rotated $\xi$ and $\zeta$ become coplanar with the hinge axis. Next, Figure $6(\mathrm{c})$, one rotates $P_{1}, \xi, \eta, \zeta$ about $\xi$ through $\omega$ to obtain $P_{1}, \xi, \eta^{\prime}, \zeta^{\prime} ; \omega$ is such that the rotated $\zeta$ becomes aligned with the hinge axis. Finally, Figure 6(d), one rotates $P_{1}, \xi, \eta^{\prime}, \zeta^{\prime}$ about $\zeta^{\prime}$ through $\psi$ to obtain $P_{1}, x_{1}, y_{1}, z_{1} ; \psi$ is such that $v_{1}$ lies in the plane defined by the rotated $\xi^{\prime}$ and $\zeta^{\prime}$.

This sequence of transformations determines the values of the angles $\phi, \omega, \psi$ for each rod. Since there are only two rod types, which will be denoted by the subscripts 1 and 2 , the three angles are

$$
\begin{aligned}
& \phi_{1}=-\phi_{2}=-\arctan \left(\tan \frac{\alpha_{1}}{2} \sin \alpha_{3}\right) \\
& \omega_{1}=-\omega_{2}=90^{\circ}+\arctan \frac{q}{\sqrt{p^{2}+r^{2}}} \\
& \psi_{1}=-\psi_{2}=\quad-180^{\circ}-\phi_{1}
\end{aligned}
$$

where

$$
\begin{aligned}
& p=\cos \alpha_{3} \tan \alpha_{3}-\sin \alpha_{3} \cos \alpha_{1} \\
& q=\sin \alpha_{3} \tan \alpha_{3}+\cos \alpha_{3} \cos \alpha_{1} \\
& r=\sin \alpha_{1}
\end{aligned}
$$

The components of the vector $v_{1}$ are, for both rod types

$$
\begin{aligned}
& a=\cos \alpha_{3} \\
& d=\sin \alpha_{3}
\end{aligned}
$$


The kinematics of the structure are defined by the variation of the hinge angles $\theta_{A}, \theta_{B}, \ldots$ These angles, see Figure 7, are defined to be zero in the folded configuration and increase monotonically during deployment. In the deployed configuration the value of $\theta_{A}$ is

$$
\theta_{A}=\arccos \left[1-2 \cos ^{2} \alpha_{3}\left(\sin \left(90^{\circ}-\frac{180^{\circ}}{n}\right)+\sin ^{2} \frac{\alpha_{1}}{2} \tan \alpha_{3}\right)^{2}\right]
$$

\subsection{Loop Closure Equation}

In the case $n=4$ the structure forms a deployable square frame. Figure 8 shows a model made from square-section timber rods connected together by "door" hinges. This is an example of structure whose rods do not have a triangular cross section. During folding, the model preserves two planes of symmetry and one 2-fold symmetry axis. This structure is an example of the well-known Bennett linkage $[\mathbf{8}, \mathbf{1 2}, \mathbf{1 3}]$.

Assuming the length of the rods, measured along the apex line, to be one unit we have

$$
v=\left[\begin{array}{l}
a \\
0 \\
d
\end{array}\right]=\left[\begin{array}{c}
\frac{\sqrt{6}}{3} \\
0 \\
\frac{\sqrt{3}}{3}
\end{array}\right]
$$

The transformation angles for rod type 1 is

$$
\begin{aligned}
& \phi_{1}=-30^{\circ} \\
& \omega_{1}=+90^{\circ}+\arctan (1 / \sqrt{8})=+109.47^{\circ} \\
& \psi_{1}=-150^{\circ}
\end{aligned}
$$

The corresponding transformation matrix is

$$
T_{1}=\left[\begin{array}{cccc}
-\frac{2}{3} & \frac{\sqrt{3}}{3} & -\frac{\sqrt{2}}{3} & \frac{\sqrt{6}}{3} \\
\frac{\sqrt{3}}{3} & 0 & -\frac{\sqrt{6}}{3} & 0 \\
-\frac{\sqrt{2}}{3} & -\frac{\sqrt{6}}{3} & -\frac{1}{3} & \frac{\sqrt{3}}{3} \\
0 & 0 & 0 & 1
\end{array}\right]
$$

and an analogous expression can be derived for rod type 2 .

The transformation matrix for a hinge with angle $\theta_{A}$ is

$$
T_{A}=\left[\begin{array}{cccc}
\cos \theta_{A} & -\sin \theta_{A} & 0 & 0 \\
\sin \theta_{A} & \cos \theta_{A} & 0 & 0 \\
0 & 0 & 1 & 0 \\
0 & 0 & 0 & 1
\end{array}\right]
$$


One can now consider a closed-loop structure consisting of 4 rods arranged in the sequence type 1 , type 2 , type 1 , type 2 and connected by revolute joints with hinge angles $\theta_{A}, \theta_{B}, \theta_{C}, \theta_{D}$. Having reached the last joint of the structure one has to ensure that it coincides with the first. More precisely, the first and last points of the linkage must be the same and the first and last revolutes must also coincide. This condition is known as the loop-closure equation $[\mathbf{8 , 9}]$.

A linkage with $n=4$ rods, i.e. two pairs of identical rods, is described by the product of two pairs of constant matrices and four angle-dependent matrices:

$$
T_{1} \times T_{\theta_{A}} \times T_{2} \times T_{\theta_{B}} \times T_{1} \times T_{\theta_{C}} \times T_{2} \times T_{\theta_{D}}=I
$$

In the general case of $n$ rods (where $n$ is even) the loop closure equation is analogous to Equation 12 , but now includes $n / 2$ pairs of constant matrices as well as $n$ angle-dependent matrices.

\subsection{Analytical Solution for $n=4$}

If symmetric behaviour is assumed, and hence $\theta_{A}=\theta_{C}$ and $\theta_{B}=\theta_{D}$, Equation 12 can be simplified to

$$
\begin{aligned}
& \left\{\left[\begin{array}{cccc}
-\frac{2}{3} & \frac{\sqrt{3}}{3} & -\frac{\sqrt{2}}{3} & \frac{\sqrt{6}}{3} \\
\frac{\sqrt{3}}{3} & 0 & -\frac{\sqrt{6}}{3} & 0 \\
-\frac{\sqrt{2}}{3} & -\frac{\sqrt{6}}{3} & -\frac{1}{3} & \frac{\sqrt{3}}{3} \\
0 & 0 & 0 & 1
\end{array}\right]\left[\begin{array}{cccc}
\cos \theta_{A} & -\sin \theta_{A} & 0 & 0 \\
\sin \theta_{A} & \cos \theta_{A} & 0 & 0 \\
0 & 0 & 1 & 0 \\
0 & 0 & 0 & 1
\end{array}\right]\right. \\
& \left.\left[\begin{array}{cccc}
-\frac{2}{3} & -\frac{\sqrt{3}}{3} & -\frac{\sqrt{2}}{3} & \frac{\sqrt{6}}{3} \\
-\frac{\sqrt{3}}{3} & 0 & \frac{\sqrt{6}}{3} & 0 \\
-\frac{\sqrt{2}}{3} & \frac{\sqrt{6}}{3} & -\frac{1}{3} & \frac{\sqrt{3}}{3} \\
0 & 0 & 0 & 1
\end{array}\right]\left[\begin{array}{cccc}
\cos \theta_{B} & -\sin \theta_{B} & 0 & 0 \\
\sin \theta_{B} & \cos \theta_{B} & 0 & 0 \\
0 & 0 & 1 & 0 \\
0 & 0 & 0 & 1
\end{array}\right]\right\}=I
\end{aligned}
$$

and solving this equation symbolically yields

$$
\theta_{B}=\arctan \frac{2 \sqrt{3} \cos ^{2} \theta_{A}-3 \sin \theta_{A} \cos \theta_{A}+3 \sin \theta_{A}-4 \sqrt{3} \cos \theta_{A}+2 \sqrt{3}}{-\cos ^{2} \theta_{A}-2 \sqrt{3} \sin \theta_{A} \cos \theta_{A}+2 \sqrt{3} \sin \theta_{A}-\cos \theta_{A}+2}
$$

A plot of $\theta_{A}$ against $\theta_{B}$ is shown in Figure 9.

\section{Numerical Solution of Loop-Closure Equation}

A general solution method for the loop closure equation is presented, based on a predictorcorrector scheme suitable for implementation in a standard Newton-Raphson iteration. For 
definiteness a loop with $n=4$ rods will be considered.

\subsection{Predictor Step}

From the closure equation in the initial configuration

$$
T_{1} T_{A_{0}} T_{3} T_{B_{0}} T_{1} T_{C_{0}} T_{3} T_{D_{0}}=I
$$

Consider small geometry changes in each hinge which do not require any deformation of the rods. For the linkage to still fit together it needs to satisfy the loop-closure equation also in the deformed configuration

$$
T_{1} T_{A} T_{3} T_{B} T_{1} T_{C} T_{3} T_{D}=I
$$

where $T_{A}, T_{B}, T_{C}$ and $T_{D}$ correspond to the general configuration defined by $\theta_{A}, \theta_{B}, \theta_{C}$ and $\theta_{D}$. Consider the Taylor expansion of the hinge angles. By ignoring higher-order terms

$$
\begin{aligned}
& \sin \left(\theta_{A_{0}}+\Delta \theta_{A_{0}}\right) \approx \sin \theta_{A_{0}}+\cos \theta_{A_{0}}\left(\Delta \theta_{A_{0}}\right) \\
& \cos \left(\theta_{A_{0}}+\Delta \theta_{A_{0}}\right) \approx \cos \theta_{A_{0}}-\sin \theta_{A_{0}}\left(\Delta \theta_{A_{0}}\right)
\end{aligned}
$$

Substituting into, e.g. the transformation matrix $T_{A}$, yields

$$
\begin{aligned}
T_{A} & =\left[\begin{array}{cccc}
\cos \left(\theta_{A_{0}}+\Delta \theta_{A_{0}}\right) & -\sin \left(\theta_{A_{0}}+\Delta \theta_{A_{0}}\right) & 0 & 0 \\
\sin \left(\theta_{A_{0}}+\Delta \theta_{A_{0}}\right) & \cos \left(\theta_{A_{0}}+\Delta \theta_{A_{0}}\right) & 0 & 0 \\
0 & 0 & 1 & 0 \\
0 & 0 & 0 & 1
\end{array}\right] \\
& \simeq T_{A_{0}}+\left[\begin{array}{cccc}
-\sin \theta_{A_{0}} & -\cos \theta_{A_{0}} & 0 & 0 \\
\cos \theta_{A_{0}} & -\sin \theta_{A_{0}} & 0 & 0 \\
0 & 0 & 0 & 0 \\
0 & 0 & 0 & 0
\end{array}\right] \Delta \theta_{A_{0}} \\
& =T_{A_{0}}+T_{A_{0}}^{\prime} \Delta \theta_{A_{0}}
\end{aligned}
$$

where $T_{A_{0}}^{\prime}$ is the derivative of $T_{A}$ calculated at $A_{0}$.

Substituting Equation 16 and analogous expressions for $T_{B}, T_{C}$, etc. into Equation 14 yields

$$
T_{1}\left(T_{A_{0}}+T_{A_{0}}^{\prime} \Delta \theta_{A_{0}}\right) T_{3}\left(T_{B_{0}}+T_{B_{0}}^{\prime} \Delta \theta_{B_{0}}\right) \cdots=I
$$


Expanding Equation 17 and rearranging, we have

$$
\begin{gathered}
T_{1} T_{A_{0}} T_{3} T_{B_{0}} T_{1} T_{C_{0}} T_{3} T_{D_{0}} \\
+\left(T_{1} T_{A_{0}}^{\prime} T_{3} T_{B_{0}} T_{1} T_{C_{0}} T_{3} T_{D_{0}}\right) \Delta \theta_{A_{0}} \\
+\left(T_{1} T_{A_{0}} T_{3} T_{B_{0}}^{\prime} T_{1} T_{C_{0}} T_{3} T_{D_{0}}\right) \Delta \theta_{B_{0}} \\
+\left(T_{1} T_{A_{0}} T_{3} T_{B_{0}} T_{1} T_{C_{0}}^{\prime} T_{3} T_{D_{0}}\right) \Delta \theta_{C_{0}} \\
+\left(T_{1} T_{A_{0}} T_{3} T_{B_{0}} T_{1} T_{C_{0}} T_{3} T_{D_{0}}^{\prime}\right) \Delta \theta_{D_{0}}=I
\end{gathered}
$$

From Equation 14, the first term of Equation 18 is equal to the identity matrix. Hence, the sum of the remaining terms must be zero. Defining $A=T_{1} T_{A_{0}}^{\prime} T_{3} T_{B_{0}} T_{1} T_{C_{0}} T_{3} T_{D_{0}}$, etc. this equation can be rearranged into

$$
A \Delta \theta_{A_{0}}+B \Delta \theta_{B_{0}}+C \Delta \theta_{C_{0}}+D \Delta \theta_{D_{0}}=[0]
$$

where [0] is a $4 \times 4$ null matrix. It is interesting to note the form of these matrices

$$
\left[\begin{array}{cccc}
0 & a_{1,2} & a_{1,3} & a_{1,4} \\
-a_{1,2} & 0 & a_{2,3} & a_{2,4} \\
-a_{1,3} & -a_{2,3} & 0 & a_{3,4} \\
0 & 0 & 0 & 0
\end{array}\right] \Delta \theta_{A_{0}}+\left[\begin{array}{cccc}
0 & b_{1,2} & b_{1,3} & b_{1,4} \\
-b_{1,2} & 0 & b_{2,3} & b_{2,4} \\
-b_{1,3} & -b_{2,3} & 0 & b_{3,4} \\
0 & 0 & 0 & 0
\end{array}\right] \Delta \theta_{B_{0}}+\cdots=[0]
$$

At first glance, the matrix equation (20) would appear to be equivalent to 9 scalar equations in 4 unknowns (not 16, since the 7 equations corresponding to the diagonal and the last row are always satisfied). The fact that the $3 \times 3$ sub-matrix in the top-left corner is skew-symmetric corresponds to the fact that any rotation can always be defined by only three elementary rotations, hence 6 of the 9 coefficients of this sub-matrix are dependent on the other 3. Hence, there remain 6 independent parameters, which form the Plucker coordinates, or line coordinates of each particular joint.

Hence, this yields a $6 \times 4$ system of equations, whose coefficient matrix is known as the Jacobian of the system [9]

$$
\left[\begin{array}{cccc}
a_{1,2} & b_{1,2} & c_{1,2} & d_{1,2} \\
a_{1,3} & b_{1,3} & c_{1,3} & d_{1,3} \\
a_{2,3} & b_{2,3} & c_{2,3} & d_{2,3} \\
a_{1,4} & b_{1,4} & c_{1,4} & d_{1,4} \\
a_{2,4} & b_{2,4} & c_{2,4} & d_{2,4} \\
a_{3,4} & b_{3,4} & c_{3,4} & d_{3,4}
\end{array}\right]\left[\begin{array}{l}
\Delta \theta_{A_{0}} \\
\Delta \theta_{B_{0}} \\
\Delta \theta_{C_{0}} \\
\Delta \theta_{D_{0}}
\end{array}\right]=\left[\begin{array}{l}
0 \\
0 \\
0 \\
0 \\
0 \\
0
\end{array}\right]
$$

If the linkage that is being analysed has mobility one, then the matrix in Equation 21 will be rank-deficient, with a rank of 3 . This implies that the equation has a single infinity of solutions 
and also indicates that the linkage has an internal mechanism. By solving Equation 21 we can find sets of $\Delta \theta^{\prime} s \neq 0$ which describe infinitesimal, zero-strain motions of the linkage.

This set of values can be obtained by computing the singular value decomposition of the Jacobian matrix $[\mathbf{1 4}, \mathbf{1 5}]$. The singular value decomposition of a matrix $A$, in general rectangular, has the expression $A=U V W^{T}$. Here, $U$ and $W$ are square, orthonormal matrices containing the leftand right-singular vectors; $V$ is a rectangular matrix with $r$ non-zero singular values on its main diagonal, where $r$ is the rank of the matrix $A$. In the present case, provided that the rank of the Jacobian matrix is 3 , the required zero-strain motion of the linkage is provided by the last column, i.e. column 4 , of the matrix $W$.

\subsection{Corrector Step}

A finite, yet small motion of the linkage where each hinge angle is changed by an amount proportional to the infinitesimal mechanism computed above is likely to induce small errors.

Let $\bar{C}$ be a configuration obtained from the predictor step, i.e. by imposing a small, and yet finite change of angles to the initial configuration. We wish to compute a configuration $C_{1}$, near $\bar{C}$, where all errors have been removed. Hence, we need to compute $\Delta C^{\prime}$, the configuration change from $\bar{C}$ to $C_{1}$.

In configuration $C_{1}$

$$
T_{1} T_{A_{1}} T_{3} T_{B_{1}} T_{1} T_{C_{1}} T_{3} T_{D_{1}}=I
$$

Although $C_{1}$ is not known, $\bar{C}$ is known. In this configuration the closure equation is not satisfied, hence one can compute an error matrix $E$ from

$$
T_{1} T_{\bar{A}} T_{3} T_{\bar{B}} T_{1} T_{\bar{C}} T_{3} T_{\bar{D}}=I+E
$$

Expanding the hinge angles in configuration $C_{1}$ in term of $\bar{C}$

$$
\theta_{A_{1}}=\theta_{\bar{A}}+\Delta \theta_{A}^{*}, \quad \theta_{B_{1}}=\theta_{\bar{B}}+\Delta \theta_{B}^{*}, \quad \cdots
$$

Now, writing the closure equation in configuration $C_{1}$, substituting a Taylor expansion for each 
hinge angle, and then manipulating the equations as above we obtain

$$
\begin{gathered}
T_{1} T_{\bar{A}} T_{3} T_{\bar{B}} T_{1} T_{\bar{C}} T_{3} T_{\bar{D}} \\
+\left(T_{1} T_{\bar{A}}^{\prime} T_{3} T_{\bar{B}} T_{1} T_{\bar{C}} T_{3} T_{\bar{D}}\right) \Delta \theta_{A}^{*} \\
+\left(T_{1} T_{\bar{A}} T_{3} T_{\bar{B}}^{\prime} T_{1} T_{\bar{C}} T_{3} T_{\bar{D}}\right) \Delta \theta_{B}^{*} \\
+\left(T_{1} T_{\bar{A}} T_{3} T_{\bar{B}} T_{1} T_{\bar{C}}^{\prime} T_{3} T_{\bar{D}}\right) \Delta \theta_{C}^{*} \\
+\left(T_{1} T_{\bar{A}} T_{3} T_{\bar{B}} T_{1} T_{\bar{C}} T_{3} T_{\bar{D}}^{\prime}\right) \Delta \theta_{D}^{*}=I
\end{gathered}
$$

From Equation 23, the first term in Equation 24 is equal to $I+E$. Hence substituting Equation 23 into Equation 24, one obtains

$$
\begin{gathered}
+\left(T_{1} T_{\bar{A}}^{\prime} T_{3} T_{\bar{B}} T_{1} T_{\bar{C}} T_{3} T_{\bar{D}}\right) \Delta \theta_{A}^{*} \\
+\left(T_{1} T_{\bar{A}} T_{3} T_{\bar{B}}^{\prime} T_{1} T_{\bar{C}} T_{3} T_{\bar{D}}\right) \Delta \theta_{B}^{*} \\
+\left(T_{1} T_{\bar{A}} T_{3} T_{\bar{B}} T_{1} T_{\bar{C}}^{\prime} T_{3} T_{\bar{D}}\right) \Delta \theta_{C}^{*} \\
+\left(T_{1} T_{\bar{A}} T_{3} T_{\bar{B}} T_{1} T_{\bar{C}} T_{3} T_{\bar{D}}^{\prime}\right) \Delta \theta_{D}^{*}=-E
\end{gathered}
$$

which can be written in the form

$$
P \Delta \theta_{A}^{*}+Q \Delta \theta_{B}^{*}+R \Delta \theta_{C}^{*}+S \Delta \theta_{D}^{*}=-E
$$

where $P=T_{1} T_{\bar{A}}^{\prime} T_{3} T_{\bar{B}} T_{1} T_{\bar{C}} T_{3} T_{\bar{D}}, \ldots$ etc. The error matrix $E$ on the right hand side has the structure

$$
\left[\begin{array}{cccc} 
& & & e_{1,4} \\
& F & & e_{2,4} \\
& & & e_{3,4} \\
0 & 0 & 0 & 0
\end{array}\right]
$$

Decompose $F$ into its symmetric and skew-symmetric components, i.e. $\left(F+F^{T}\right) / 2,\left(F-F^{T}\right) / 2$, and consider only the skew-symmetric part, whose coefficients are denoted by $\bar{e}_{1,2}, \bar{e}_{1,3}$ and $\bar{e}_{2,3}$. This transformed equation can be treated in the same way as Equation 20, thus rearranging it into 6 scalar equations

$$
\left[\begin{array}{llll}
p_{1,2} & q_{1,2} & r_{1,2} & s_{1,2} \\
p_{1,3} & q_{1,3} & r_{1,3} & s_{1,3} \\
p_{2,3} & q_{2,3} & r_{2,3} & s_{2,3} \\
p_{1,4} & q_{1,4} & r_{1,4} & s_{1,4} \\
p_{2,4} & q_{2,4} & r_{2,4} & s_{2,4} \\
p_{3,4} & q_{3,4} & r_{3,4} & s_{3,4}
\end{array}\right]\left[\begin{array}{c}
\Delta \theta_{A}^{*} \\
\Delta \theta_{B}^{*} \\
\Delta \theta_{C}^{*} \\
\Delta \theta_{D}^{*}
\end{array}\right]=-\left[\begin{array}{l}
\bar{e}_{1,2} \\
\bar{e}_{1,3} \\
\bar{e}_{2,3} \\
e_{1,4} \\
e_{2,4} \\
e_{3,4}
\end{array}\right]
$$


The least squares solution of Equation 27 is used to determine the minimal correcting angles $\Delta \theta^{*}$ due to the errors in configuration $\bar{C}$

$$
\Delta \theta^{*}=-\sum_{i=1}^{r} \frac{w_{i} u_{i}^{T}}{v_{i, i}} e
$$

where $U V W^{T}$ is the singular value decomposition of the $6 \times 4$ coefficient matrix in Equation 27 and $r$ is the number of non-zero singular values. Also, $w_{i}$ is the $i^{t h}$ column of matrix $W ; u_{i}$ is the $i^{t h}$ column of $U ; v_{i, i}$ is the $i, i$ term in matrix $V ;-e$ is the vector on the right hand side of Equation 27. Note that the singular value decomposition of the Jacobian matrix in Equation 21 is often a good approximation for evaluating Equation 28.

\section{Results}

The predictor-corrector algorithm presented in Section 4 has been used to simulate the deployment of two closed-loop structures of the type described in Section 3. In each simulation, the structure was moved along its kinematic path in 200 steps, starting from the fully-folded configuration, until the hinge rotation $\theta_{A}$ reached the value corresponding to the fully deployed configuration, computed from Equation 10.

First, the behaviour of Hedgepeth's deployable square structure, $n=4$, was analysed, but this time without assuming symmetric behaviour. Since it is already known from Section 3.2 that this structure has a symmetric motion path, relaxing the symmetry constraint may lead to the discovery of alternative, symmetry-breaking motion paths.

The results of the simulation are shown in Figures 10 and 11. Figure 10 shows a plot of the variation of 3 hinge angles in terms of a chosen hinge angle, $\theta_{A}$. Note that $\theta_{B}=\theta_{D}$ and $\theta_{C}=\theta_{A}$ at all stages, hence confirming that the structure remains symmetric at all stages. In other words, there are no symmetry-breaking alternative paths. Therefore, the behaviour predicted by the numerical simulation is identical to the analytical results plotted in Figure 9. Six snapshots from the deployment sequence are shown in Figure 11.

Next, the behaviour of the Hedgepeth deployable hexagon, $n=6$, was analysed. For this structure it is possible to show the existence of a 3 -fold symmetric deployment path that allows the structure to move continuously from its folded, bundle configuration to the deployed, hexagonal configuration [4]. Without making any assumptions, our numerical simulation has produced the results shown in Figure 12 and 13. The plot in Figure 12 shows that also in this case there is a 
unique motion path, with $\theta_{A}=\theta_{C}=\theta_{E}$ and $\theta_{B}=\theta_{D}=\theta_{F}$ throughout. Six snapshots from the simulation are shown in Figure 13.

A key issue, when considering the behaviour of a deployable structure, is whether there is the possibility of it deploying into the "wrong shape". This issue can be investigated by monitoring the singular values of the Jacobian matrix at every step of the deployment simulation, and particularly the ratio between the largest and smallest non-zero singular values. The smallness of this ratio is related to the sensitivity of the real structure to the presence of small geometric imperfections or to small elastic deformation of its members.

In the two examples that have been presented in this section this investigation leads to the conclusion that both structures are insensitive to errors, and hence "robust". However, this is not always the case and an example will be presented in the next section.

\section{A Further Example}

The examples presented in the previous section, which have been shown to have unique deployment paths, are not typical of over-determinate closed-loop linkages. Our search for deployable rectangular structures, of which an example was shown in Figure 3, has produced many examples of structures with mobility higher than one in at least one intermediate configuration. The full story will be presented elsewhere but here we show an example that highlights the potential usefulness of the proposed algorithm in detecting any special configurations where the kinematic path can bifurcate.

Figure 14 shows a particular realization of an over-constrained linkage proposed by S.D. Guest [16]. This model is made from square section timber rods forming a "picture frame" similar to that in Figure 8, but in this particular case the structure is of rectangular shape. The arrangement of the rods is similar to that in Figure 8 as, again, one set of cross-sectional diagonals lie in the central plane of the structure and all four corners have been cut at $45^{\circ}$ to the centre lines of the rods. However, this time the two shorter sides have been cut in the middle. Six "door" hinges have been attached to the upward facing faces of this structure: the corner hinges face inwards, whereas the mid-side hinges face outwards.

Like the structures discussed in Section 5, this structure also has a rank-deficient Jacobian matrix and its mobility is initially one. We have used our path-tracing algorithm to simulate its motion and, unlike the examples in Section 5, we have found that here the rank of the Jacobian matrix 
drops from 5 to 4 in a particular, intermediate configuration. Hence, the deployment path of this structure has a symmetry-breaking bifurcation point, corresponding to the vanishing of the 5th singular value of the Jacobian matrix. In this configuration it is possible for the structure to switch to an alternative deployment path, as illustrated in Figure 15.

\section{Discussion}

This paper has presented a general methodology for carrying out high accuracy kinematic simulations of closed-loop linkages. A particular challenge associated with the linkages that are of current interest for applications in deployable structures is that they typically contain six revolute joints and hence, in order to have mobility, they have to be over-constrained. Yet they are required to have a unique deployment path and so the Jacobian matrix associated with these linkages should ideally be rank-deficient with nullity of one.

The proposed algorithm has been used to analyse three deployable structures that have the special property of folding into a tight bundle and deploying into a flat polygonal shape. The first two structures satisfy all of the conditions listed above, and yet their their deployment kinematics involve a complex motion of the rods relative to one another. This limits the range of applications for which these structures can be used; for example, if a thin, flat membrane were to be attached to either of these structures, during deployment it would be required to stretch by a significant amount. The third structure would not have this problem, but has been shown that its deployment path has a bifurcation point. Using the tools presented in this paper, we have a carried out an extensive study of alternative linkages; the results will be published shortly [5].

\section{Acknowledgements}

The inspiration for the work presented in this paper was provided by the late Dr John M. Hedgepeth. We thank Dr M.M. Mikulas and Dr T. Murphey for help in making a physical model based on Hedgepeth's work. A preliminary version of this paper was presented at the 44th SDM Conference [17]. Helpful comments on the presentation of this paper by two anonymous reviewers are acknowledged. 


\section{References}

1 Mikulas, M. Personal communication.

2 Crawford, R.F., Hedgepeth, J.M. and Preiswerk, P.R. Spoked wheels to deploy large surfaces in space: weight estimates for solar arrays. NASA-CR-2347, 1973.

3 Bennett, G.T. A new mechanism. Engineering, 1903, 76, 777-778.

4 Chen, Y., You, Z. and Tarnai, T. Threefold-symmetric Bricard linkages for deployable structures. International Journal of Solids and Structures, 2005, 42, 2287-2301.

5 Gan, W.W. Analysis and design of closed-loop deployable structures. PhD Dissertation, University of Cambridge, in preparation.

6 Hoberman, C. Reversibly expandable structures. USA Patent No. 4981732, 1991.

7 Pellegrino, S. and You, Z. Cable-stiffened pantographic deployable structures. Part 2: Mesh Reflector. AIAA Journal, 1997, 35(8), 1348-1355.

8 Waldron, K.J., and Kinzel, G.L. Kinematics, dynamics, and design of machinery, Second Edition, John Wiley \& Sons, Danvers, MA, 2004.

9 Uicker, J.J., Jr, Pennock, G.R., and Shigley, J.E. Theory of machines and mechanisms, third edition, Oxford University Press, New York, 2003.

10 Denavit, J. and Hartenberg, R.S. A kinematic notation for lower-pair mechanisms based on matrices, Journal of Applied Mechanics, June 1955, 215-221.

11 Goldstein, H. Classical Mechanics, Second Edition. Addison-Wesley, Reading, MA, 1980.

12 Chen, Y. Design of structural mechanisms. PhD Dissertation, University of Oxford, 2003.

13 Hunt, K.H. Kinematic geometry of mechanisms, Oxford University Press, 1978.

14 Strang, G. Linear Algebra and its Applications, Thomson Learning, 1988.

15 Kumar, P., and Pellegrino, S. Computation of kinematic paths and bifurcation points. International Journal of Solids and Structures, 2000, 37(46-47), 7003-7027.

16 Pellegrino, S., Green, C., Guest, S.D. and Watt, A. SAR advanced deployable structure, Technical Report CUED/D-STRUCT/TR191, Department of Engineering, University of Cambridge, 2000. 
17 Gan, W.W., and Pellegrino, S. Closed-Loop Deployable Structures. In: Proceedings of 44th AIAA/ASME/ASCE/AHS/ASC Structures, Structural Dynamics and Materials Conference, 7-10 April 2003, Norfolk, VA, AIAA 2003-1450.

\section{Main Notation}

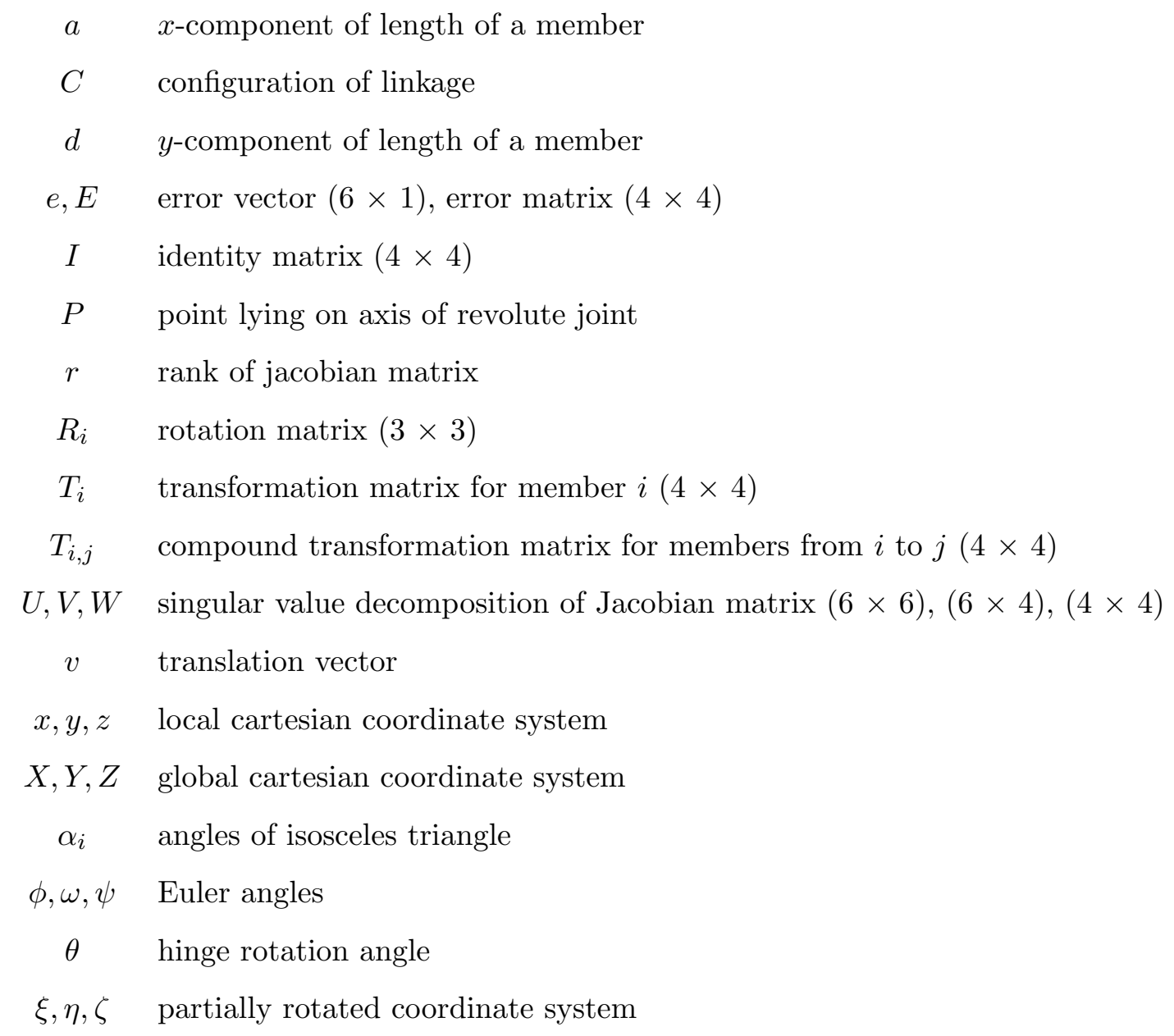

Subscripts:

0 initial configuration

Superscripts:

* correction

$\overline{(~)}$ incremented configuration 


\section{List of Figures}

1 Folded and deployed configurations of Hoop-Column Antenna demonstrator (courtesy of Toshiba Corporation). . . . . . . . . . . . . . . . . . . 18

2 Ten-sided closed loop deployable structure proposed by J. Hedgepeth [2]. . . . . 19

3 Deployable 6 -bar rectangular structure $($ from $[5]) \ldots \ldots \ldots \ldots$

4 Transformation of coordinate system $O, x, y, z$ to $P_{1}, x_{1}, y_{1}, z_{1}$ and then to $P_{2}, x_{2}, y_{2}, z_{2}$. 21

$5 \quad$ Geometry of Hedgepeth's deployable structure (a) folded (only rods 1 and 2 are shown) and (b) deployed. . . . . . . . . . . . . . . . . . . 22

6 Elementary transformations between coordinate systems for a rod. . . . . . . . . 23

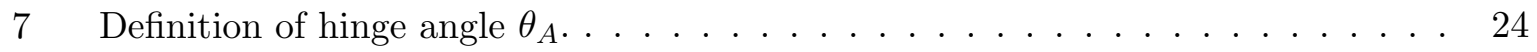

8 Deployable square frame proposed by Hedgepeth. . . . . . . . . . . . . . . 25

$9 \quad \theta_{A}$ vs $\theta_{B}$ for Hedgepeth's deployable square structure (having assumed symmetric

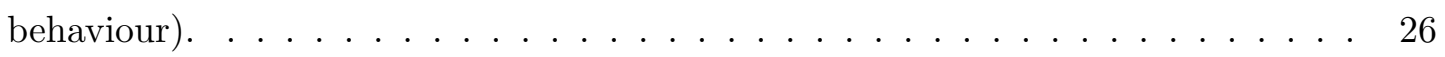

10 Variation of hinge angles during deployment of 4-rod linkage. . . . . . . . . . . 27

11 Deployment sequence of 4 -rod linkage. . . . . . . . . . . . . . . . 28

12 Variation of hinge angles during deployment of 6-rod linkage. . . . . . . . . . 29

13 Deployment sequence of 6 -rod linkage. . . . . . . . . . . . . . . . . 30

14 Deployable rectangular frame proposed by S.D. Guest. . . . . . . . . . . . . . . 31

15 Variation of singular values during deployment of Guest's rectangular frame. . . 32

16 Symmetry-breaking path of Guest's rectangular frame. . . . . . . . . . . . . . . 32 

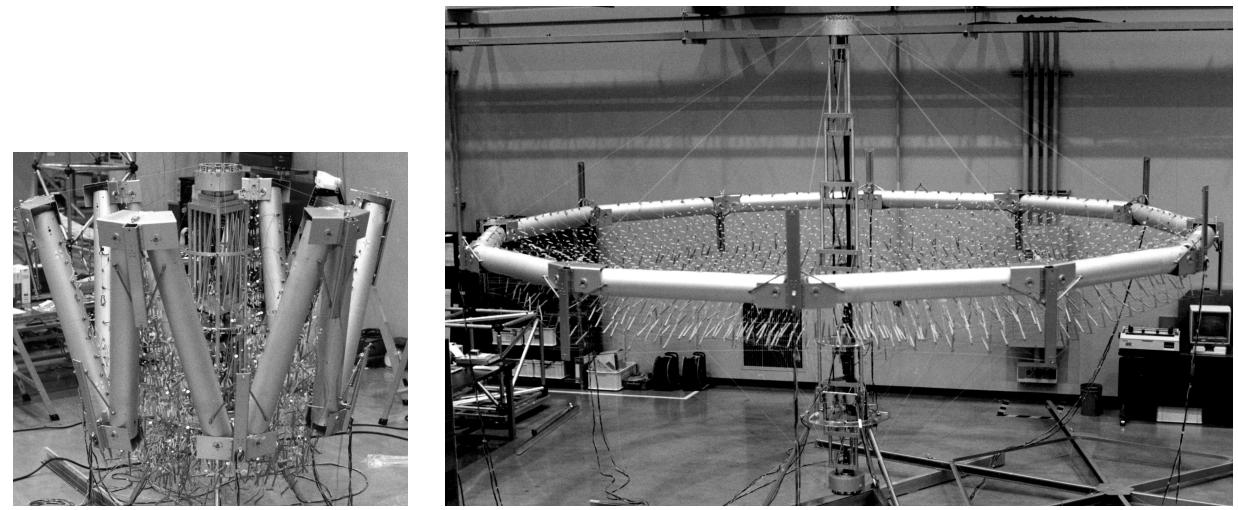

Figure 1: Folded and deployed configurations of Hoop-Column Antenna demonstrator (courtesy of Toshiba Corporation). 

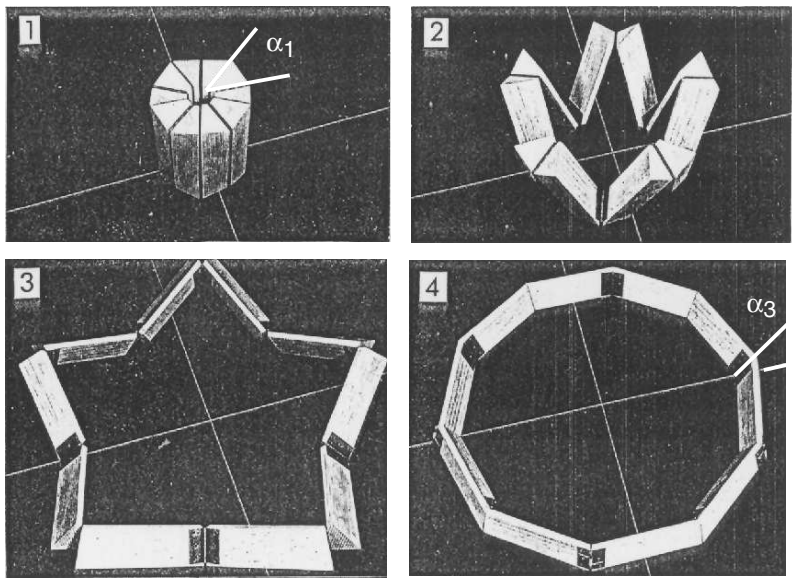

Figure 2: Ten-sided closed loop deployable structure proposed by J. Hedgepeth [2]. 

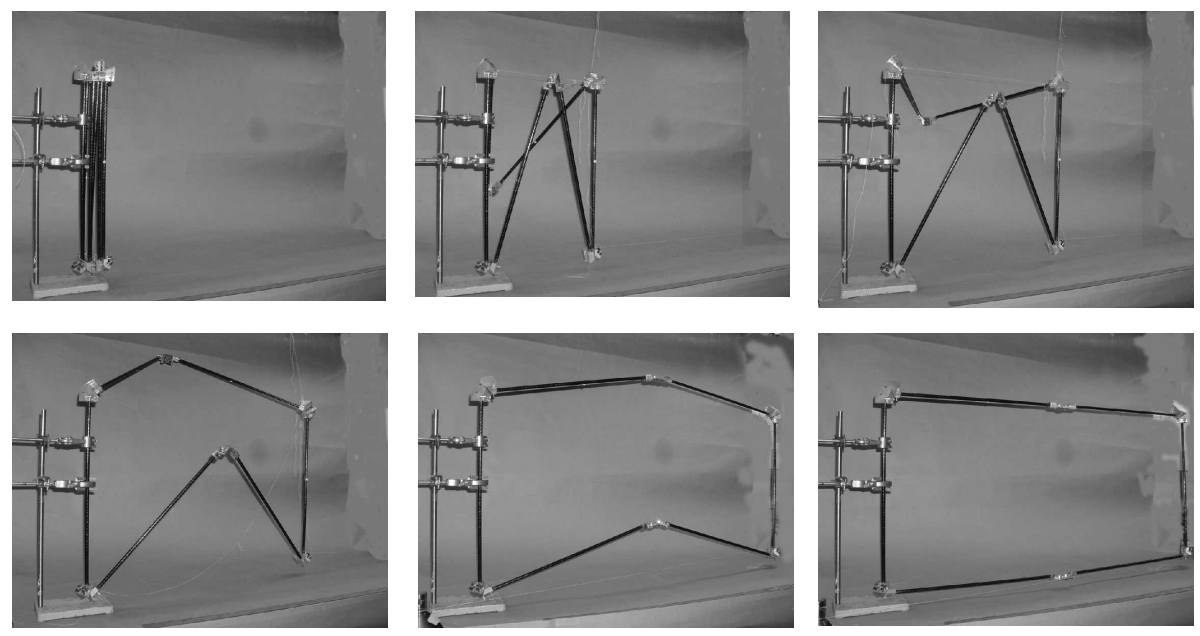

Figure 3: Deployable 6-bar rectangular structure (from [5]). 


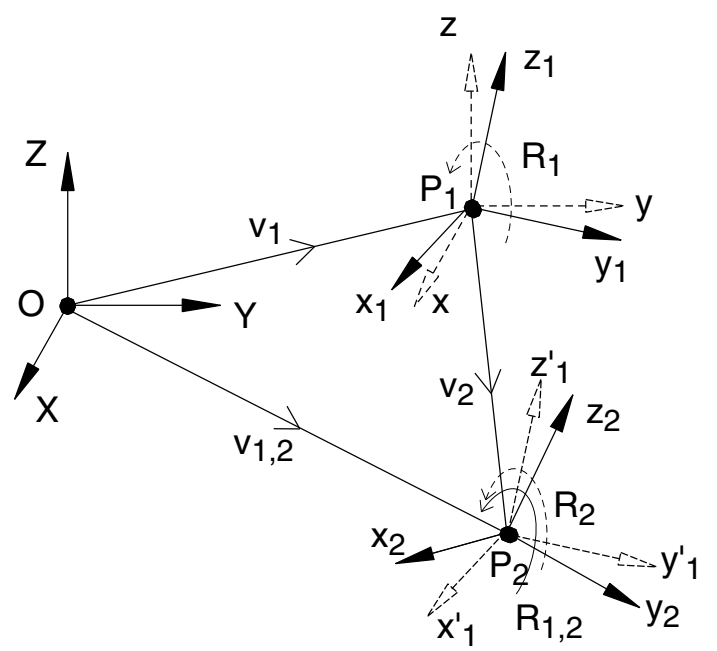

Figure 4: Transformation of coordinate system $O, x, y, z$ to $P_{1}, x_{1}, y_{1}, z_{1}$ and then to $P_{2}, x_{2}, y_{2}, z_{2}$. 


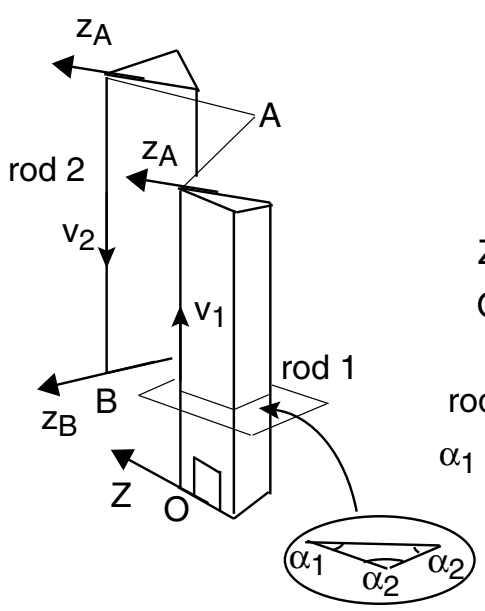

(a)

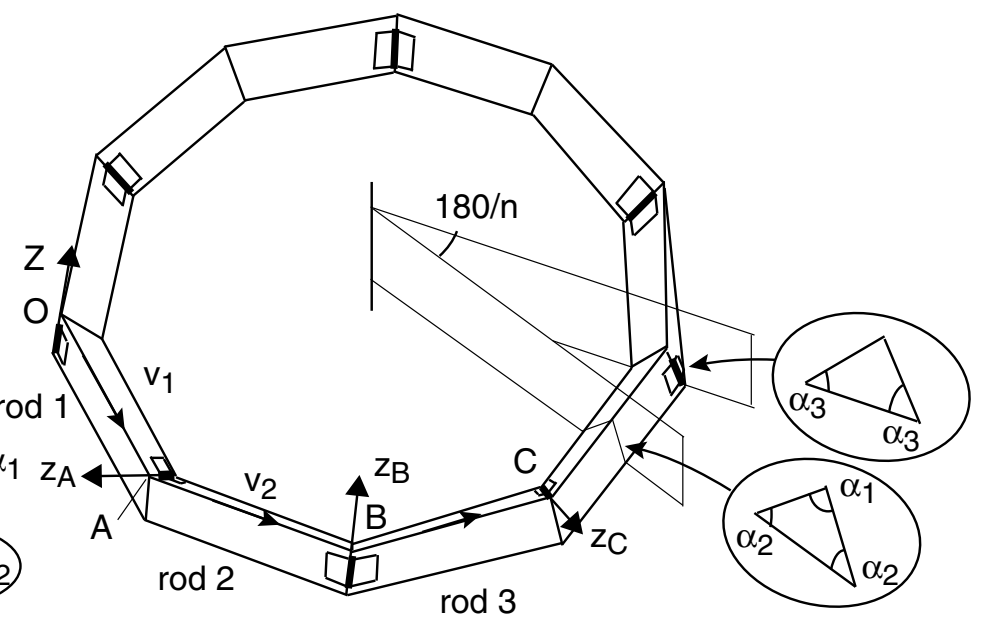

(b)

Figure 5: Geometry of Hedgepeth's deployable structure (a) folded (only rods 1 and 2 are shown) and (b) deployed. 
(a)

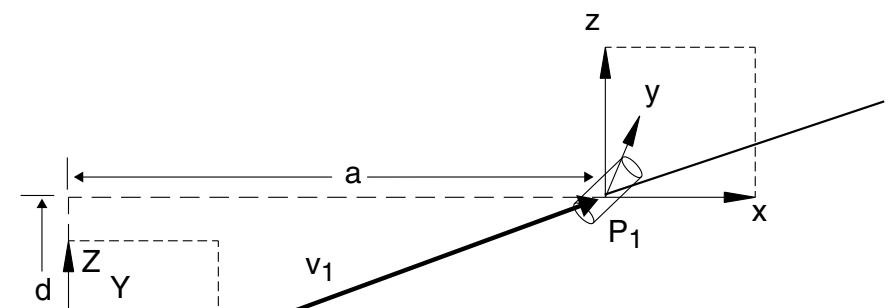

(b)
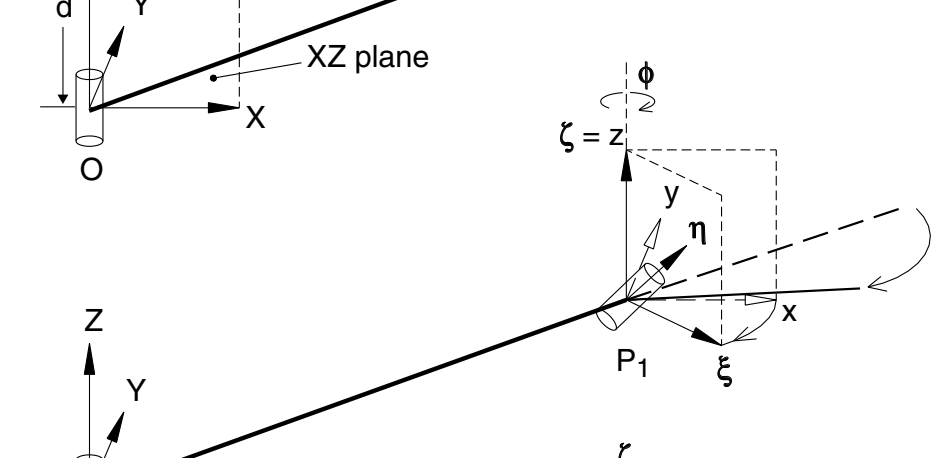

(c)
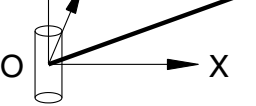

(c)

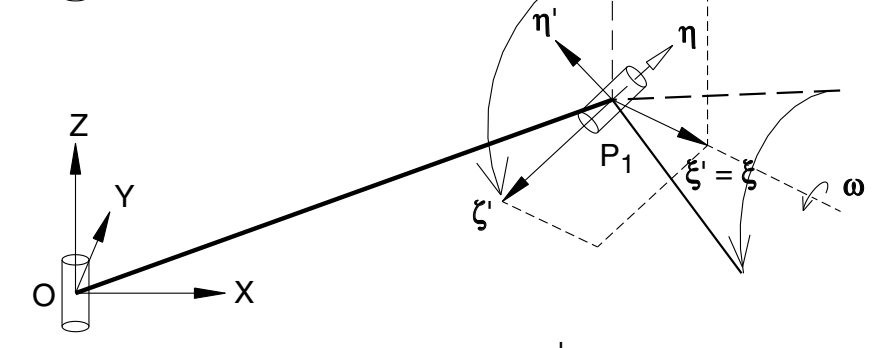

(d)

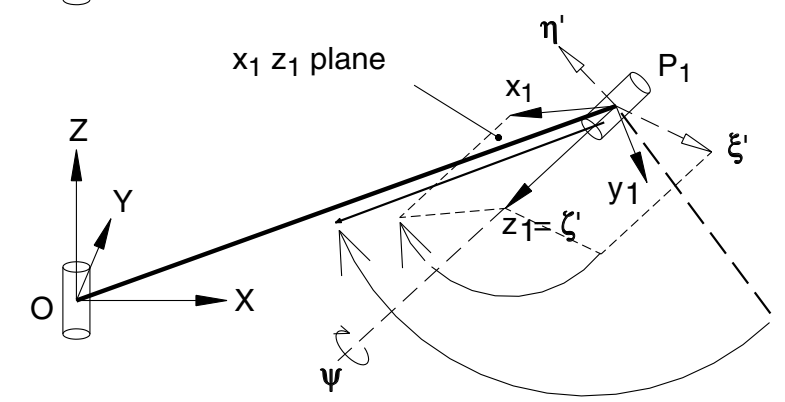

Figure 6: Elementary transformations between coordinate systems for a rod. 


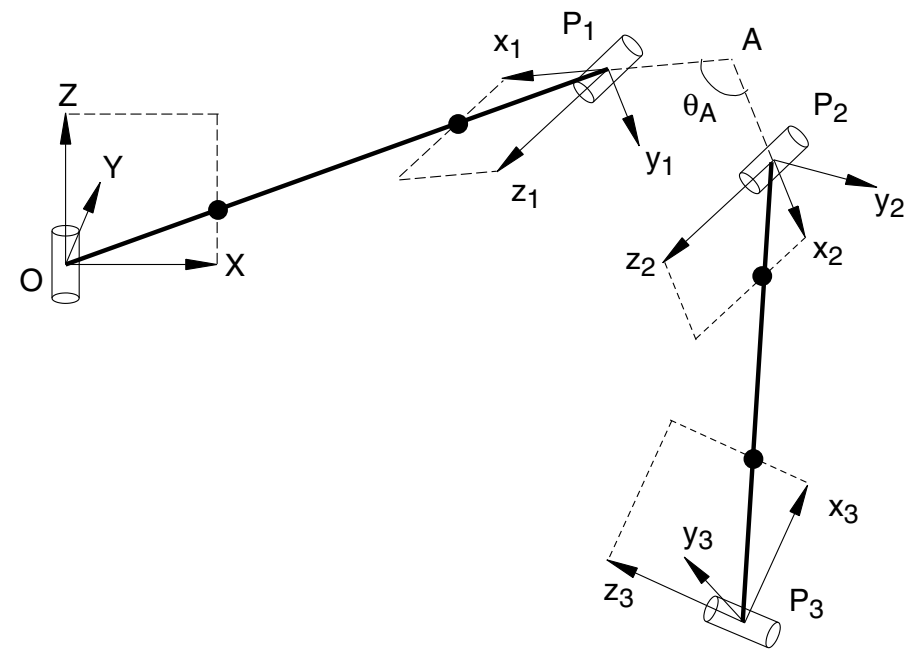

Figure 7: Definition of hinge angle $\theta_{A}$. 


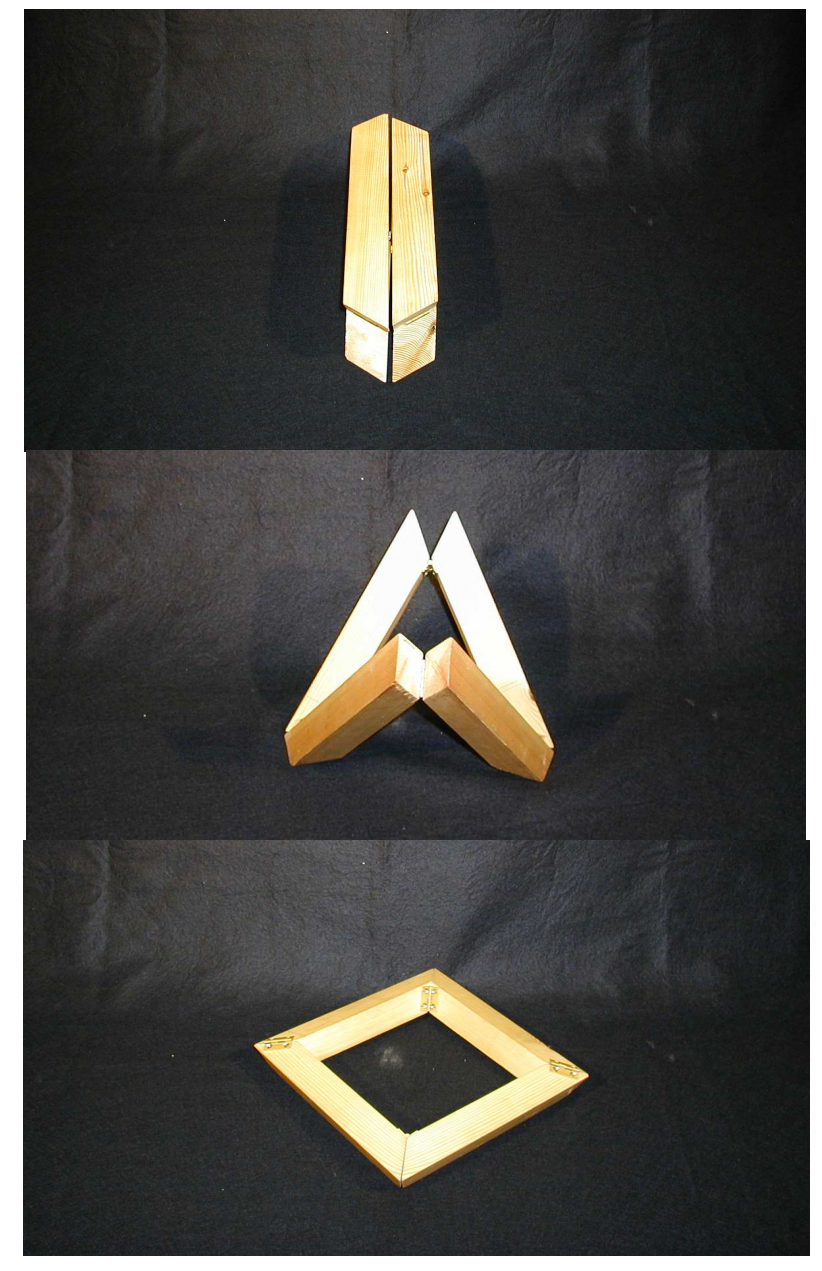

Figure 8: Deployable square frame proposed by Hedgepeth. 


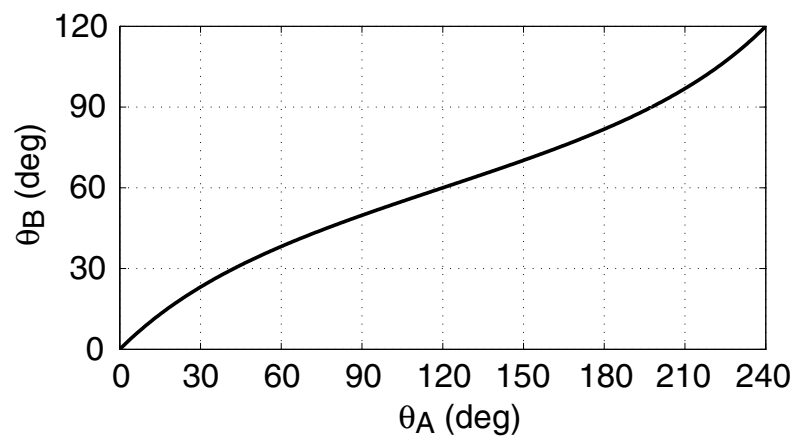

Figure 9: $\theta_{A}$ vs $\theta_{B}$ for Hedgepeth's deployable square structure (having assumed symmetric behaviour). 


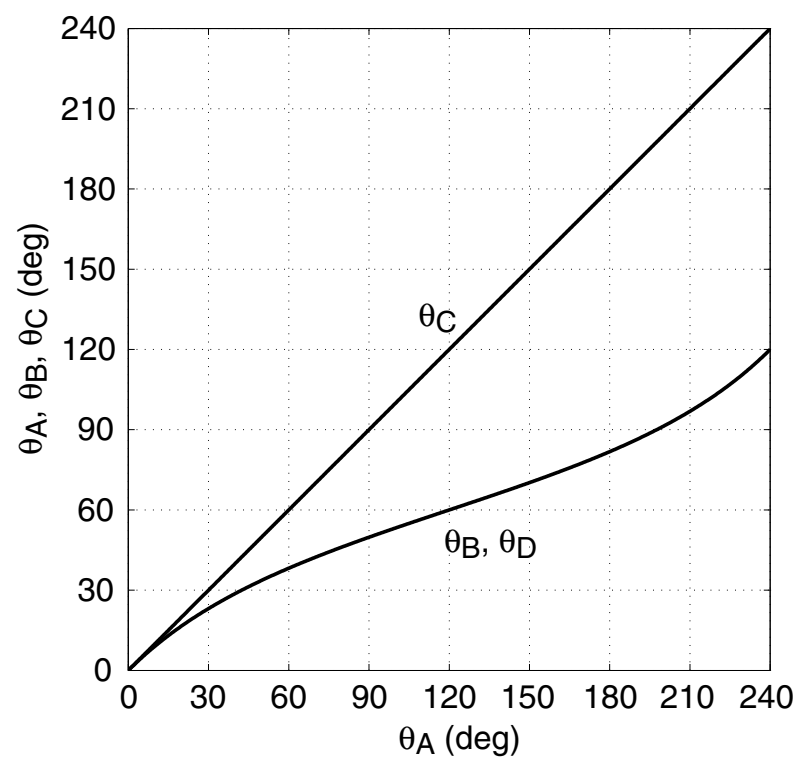

Figure 10: Variation of hinge angles during deployment of 4-rod linkage. 

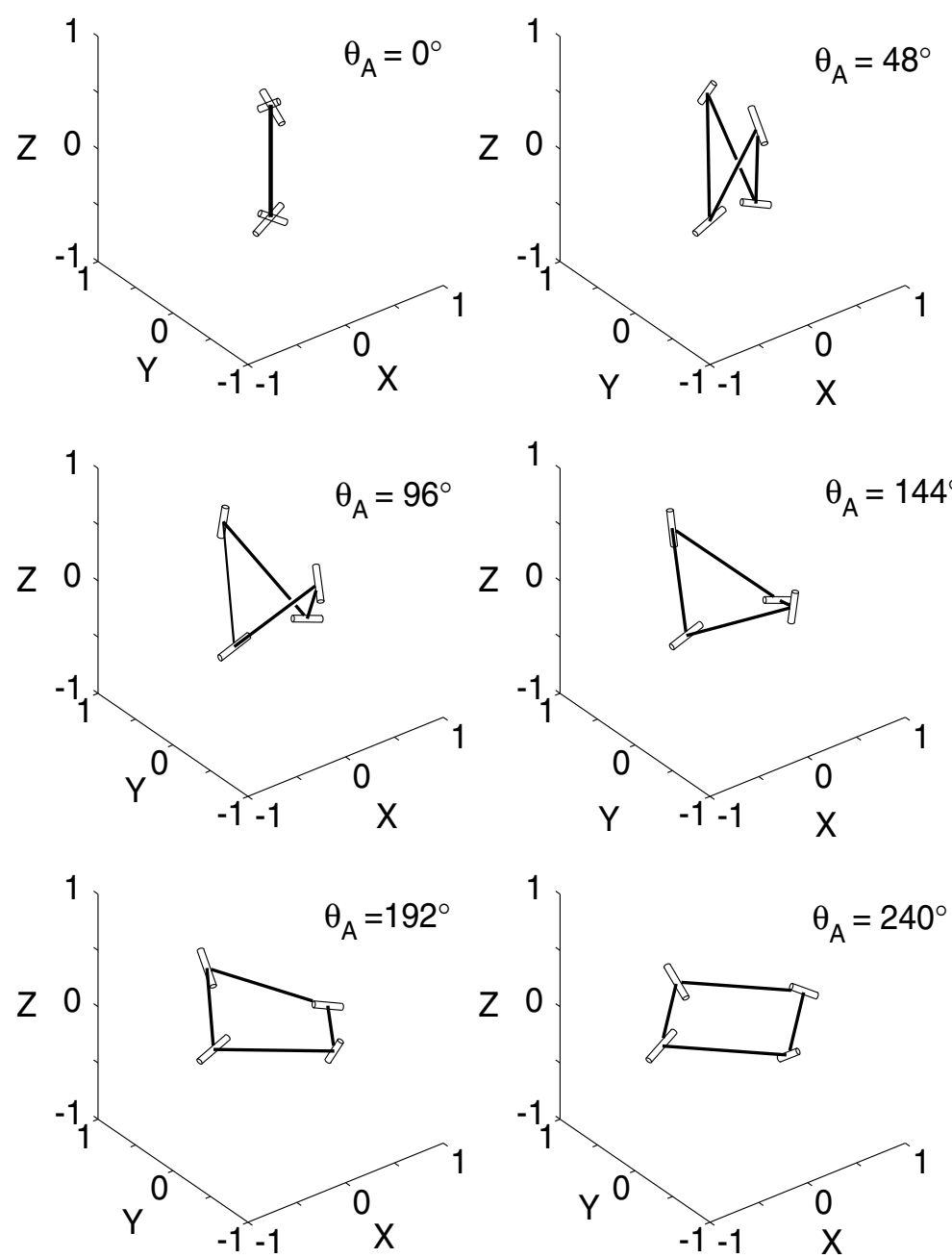

Figure 11: Deployment sequence of 4-rod linkage. 


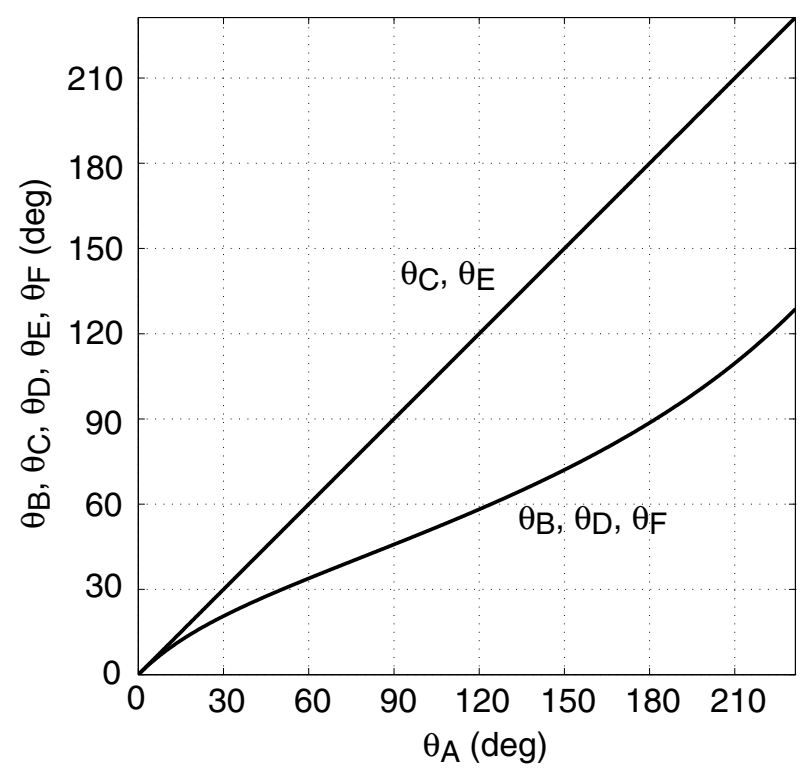

Figure 12: Variation of hinge angles during deployment of 6-rod linkage. 

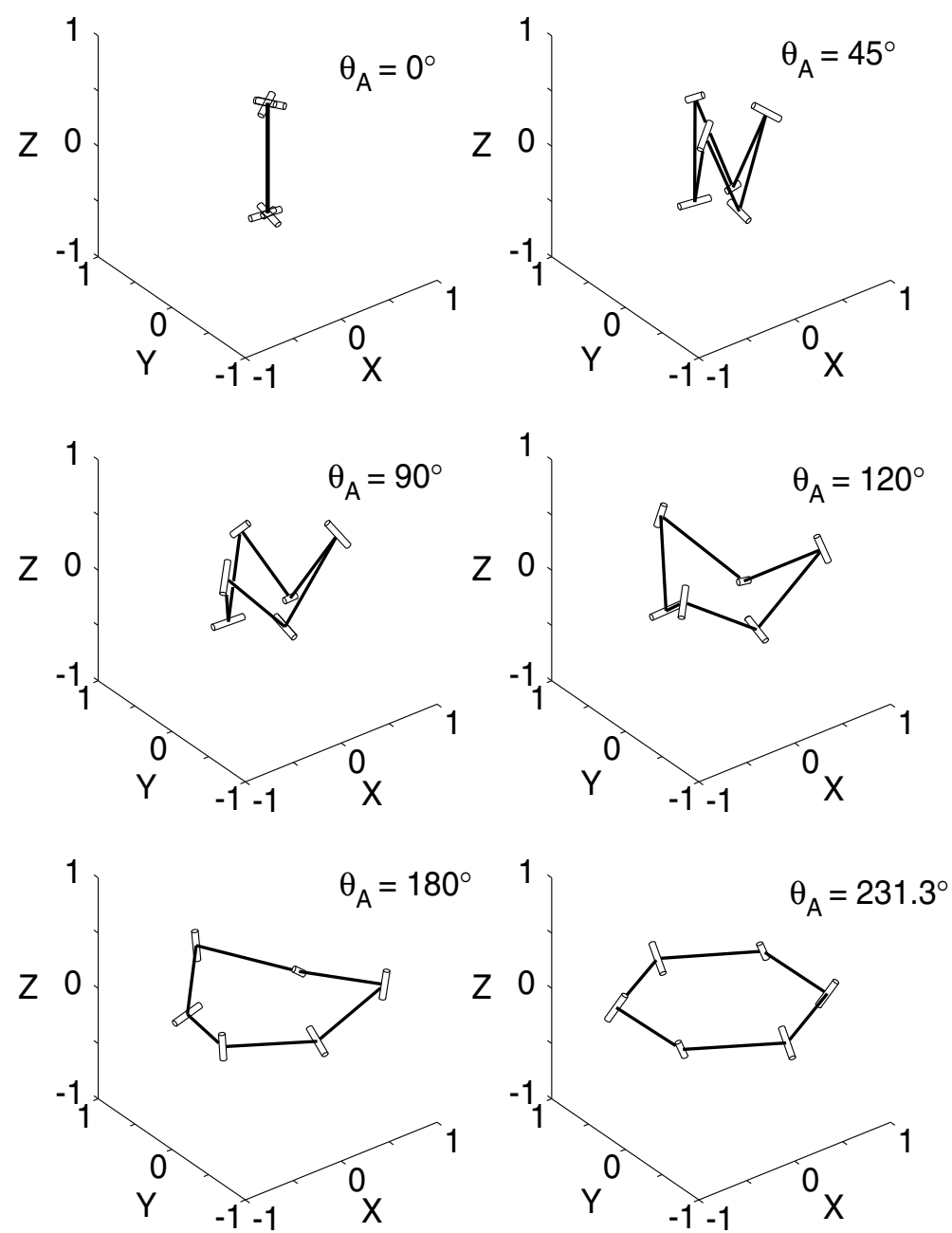

Figure 13: Deployment sequence of 6-rod linkage. 


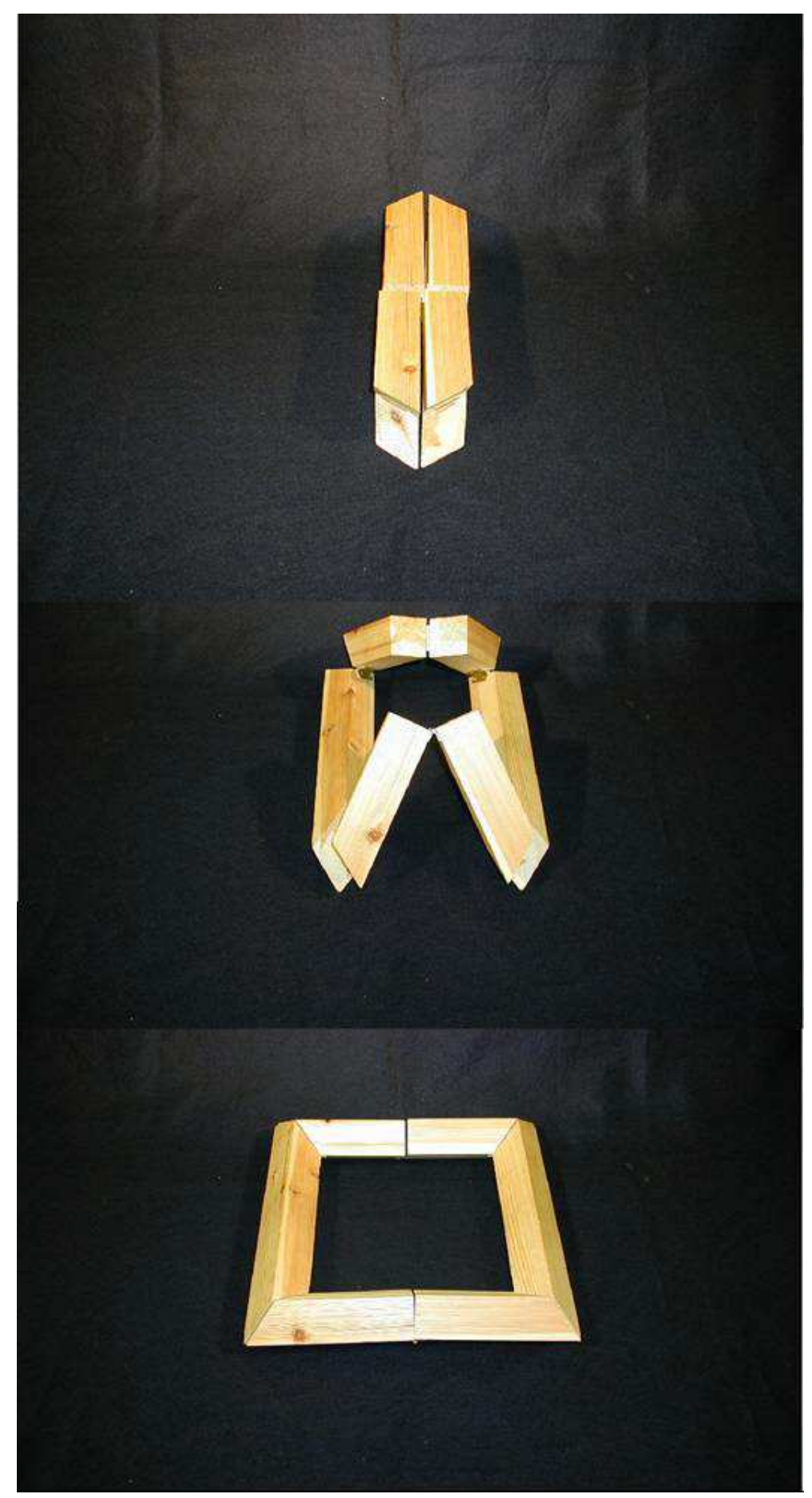

Figure 14: Deployable rectangular frame proposed by S.D. Guest. 


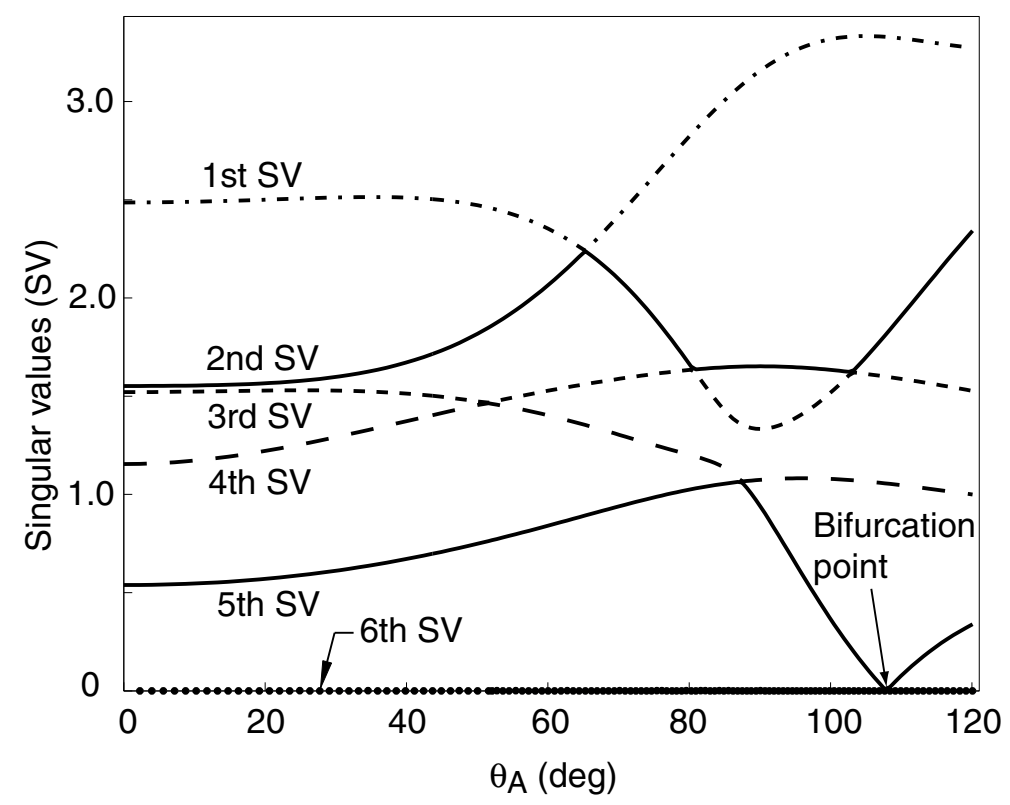

Figure 15: Variation of singular values during deployment of Guest's rectangular frame.

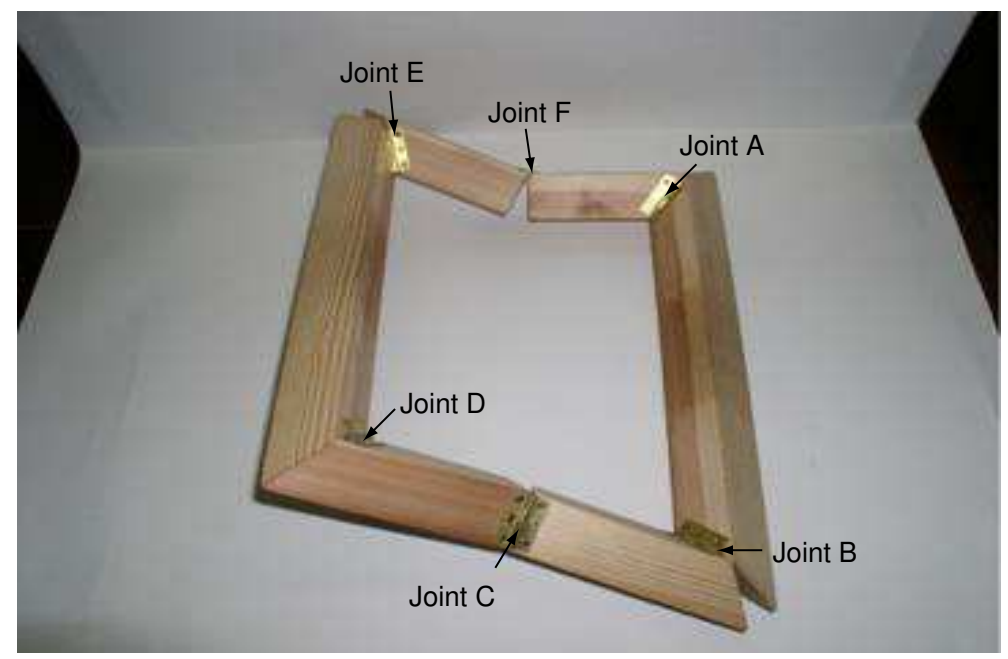

Figure 16: Symmetry-breaking path of Guest's rectangular frame. 